\title{
Discours
}

Revue de linguistique, psycholinguistique et

informatique. A journal of linguistics, psycholinguistics

and computational linguistics

$17 \mid 2015$

Varia

\section{Contraintes sur le discours et genre de discours contraint : le commentaire sportif télévisé en direct}

Catherine Mathon, Gilles Boyé, Sandra Augendre et Anna Kupść

\section{OpenEdition}

Journals

Édition électronique

URL : http://journals.openedition.org/discours/9082

DOI : $10.4000 /$ discours.9082

ISSN : 1963-1723

Éditeur :

Laboratoire LATTICE, Presses universitaires de Caen

\section{Référence électronique}

Catherine Mathon, Gilles Boyé, Sandra Augendre et Anna Kupść, « Contraintes sur le discours et genre de discours contraint : le commentaire sportif télévisé en direct », Discours [En ligne], 17 | 2015, mis en ligne le 22 décembre 2015, consulté le 30 avril 2019. URL : http://journals.openedition.org/ discours/9082 ; DOI : 10.4000/discours.9082

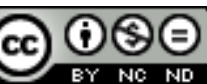

Discours est mis à disposition selon les termes de la licence Creative Commons Attribution - Pas d'Utilisation Commerciale - Pas de Modification 4.0 International. 

Revue de linguistique, psycholinguistique et informatique

\title{
Contraintes sur le discours et genre de discours contraint : le commentaire sportif télévisé en direct
}

\author{
Catherine Mathon \\ CLLE-ERSSàB (UMR 5263) \\ Université Bordeaux Montaigne \\ Gilles Boyé \\ CLLE-ERSSàB (UMR 5263) \\ Université Bordeaux Montaigne \\ Sandra Augendre \\ CLLE-ERSSàB (UMR 5263) \\ Université Bordeaux Montaigne \\ Anna Kupść \\ CLLE-ERSSàB (UMR 5263) \\ Université Bordeaux Montaigne
}

\begin{abstract}
Catherine Mathon, Gilles Boyé, Sandra Augendre et Anna Kupść, «Contraintes sur le discours et genre de discours contraint: le commentaire sportif télévisé en direct», Discours [En ligne], 17 | 2015, mis en ligne le 22 décembre 2015.
\end{abstract}

URL: http://discours.revues.org/9082 



\title{
Contraintes sur le discours et genre de discours contraint : le commentaire sportif télévisé en direct
}

\author{
Catherine Mathon \\ CLLE-ERSSàB (UMR 5263) \\ Université Bordeaux Montaigne \\ Gilles Boyé \\ CLLE-ERSSàB (UMR 5263) \\ Université Bordeaux Montaigne \\ Sandra Augendre \\ CLLE-ERSSàB (UMR 5263) \\ Université Bordeaux Montaigne \\ Anna Kupść \\ CLLE-ERSSàB (UMR 5263) \\ Université Bordeaux Montaigne
}

Nous présentons dans cet article une proposition d'analyse d'un genre de discours oral, le commentaire sportif télévisé en direct. Notre travail se démarque des précédentes études sur le commentaire sportif en ce qu'il propose une analyse à différents niveaux: prosodique, syntaxique et à terme, lexical. Par ailleurs, nous nous démarquons également des études antérieures sur les genres de discours, y compris les genres oraux, en prenant en compte les contraintes extralinguistiques qui pèsent sur la production du discours. L'intégration des contraintes extralinguistiques se fait dans le cadre de la théorie de l'optimalité appliquée ici au discours. Cette intégration nous amène à proposer une analyse et une annotation à différents niveaux, annotation qui inclut celle du contenu commenté, c'est-à-dire des images du sport telles qu'elles sont diffusées, de manière ensuite à les mettre en relation avec le commentaire.

Mots clés: genre de discours, commentaire sportif en direct, contraintes extralinguistiques, théorie de l'optimalité, analyse syntaxique, analyse prosodique

The paper presents an analysis of a specific oral discourse genre, namely live TV sports commentary. The specificity of our work, with respect to previous studies of this genre, is that our proposal takes into account several linguistic aspects simultaneously: prosodic, syntactic and, at a long-term, lexical. Our approach differs also from other studies of discourse genres as not only do we integrate the extra-linguistic constraints into our analysis but also we consider them driving factors for linguistic production of the commentary. Ou approach is formalised in terms of Optimality Theory applied to discourse analysis. In order to illustrate our proposal, we provide a detailed annotation of our video corpus of rugby match in terms of game actions and only then do we align them with and analyse linguistic content of the corresponding commentary.

Keywords: genre of discourse, live TV sports commentary, extra-linguistic constraints, Optimality Theory, syntactic analysis, prosodic analysis 


\section{Introduction}

Le présent article propose de poser les bases nécessaires à une description linguistique d'un genre de discours oral (phonogenre) particulier, le commentaire sportif télévisé en direct, à partir d'un corpus de commentaires de matchs de rugby (Lortal et Mathon, 2008). Nous entendons par "genre», un type de discours caractérisé entre autres choses par les conditions de production de ce discours («traits situationnels», Pršir et al., 2013; Goldman et al., 20II), et nous appelons «style», les productions linguistiques représentatives de ce discours. Notre étude s'inscrit dans la suite d'une série de travaux récents qui se sont concentrés soit sur les aspects discursifs et syntaxiques propres au genre (Deulofeu, 2000; Brès, 2007; Krazem, 20II ; Augendre et Mathon, 20I2a et b), soit sur les aspects prosodiques (Mathon et Boulakia, 2009; Audrit et al., 2012; Pršir et al., 2013). Une partie de ces travaux portant essentiellement sur l'analyse de matchs de football (Deulofeu, 2000 et Krazem, 2oII, notamment), il nous semblait intéressant de faire varier le sport, afin de déterminer si les mêmes structures s'appliquaient. Le rugby présentant des caractéristiques similaires au football, en ceci qu'il s'agit d'un sport qui oppose deux équipes qui se disputent un ballon sur une durée de jeu proche, nous a paru un bon objet d'étude. Nous avons constaté que malgré les similitudes entre les deux sports, les structures linguistiques présentes dans le commentaire s'avéraient effectivement différentes. L'effet est encore plus significatif si l'on compare un match et un autre type de sport, comme par exemple une course (Augendre et al., 2015, soumis).

Nous proposons une analyse multimodale, qui prend en compte les choix lexicaux, les constructions syntaxiques et les phénomènes prosodiques caractéristiques du genre, pour les relier aux contraintes de production du discours. Le commentaire sportif en direct présente en effet des conditions de production du discours particulières dont nous supposons qu'elles contraignent fortement le discours. En plus de la dimension orale qui doit être prise en compte dans l'analyse discursive, le commentaire sportif en direct est également fortement marqué par la dimension visuelle (nous travaillons sur des commentaires sportifs diffusés à la télévision). En effet, une partie du commentaire sportif consiste à décrire/narrer les actions sportives qui se déroulent sur le terrain, actions dont les images sont sélectionnées et diffusées en direction du public de téléspectateurs. Ces actions ne sont que partiellement prévisibles par le commentateur qui doit constamment adapter son discours à ce qui se passe sur le terrain. Cette «adaptation» passe par une série de choix et d'évaluations:

- choix des actions importantes qui doivent impérativement être commentées;

- évaluation des possibles suites d'actions;

- évaluation du temps disponible pour le commentaire;

- choix des structures syntaxiques et lexicales les plus efficaces pour un commentaire au plus près (sans un décalage temporel trop important) du visuel. 
Nous cherchons donc à mettre en relation ces contraintes de production du discours, liées au sport commenté, les contraintes du genre (commentaire sportif, télévisé, en partie synchrone avec l'action commentée) et les réalisations linguistiques et prosodiques des commentateurs. Nous cherchons également à mettre en évidence les variations à l'intérieur du genre, en tenant compte:

- des changements de locuteur en fonction de la nature des phases de jeu commentées, du rythme et du type de commentaire fourni, partant du principe que les différents locuteurs qui participent au commentaire ne possèdent pas les mêmes compétences linguistiques;

- des variations liées à la nature de l'événement sportif commenté; on suppose, par exemple, qu'un match de football et un match de rugby présentent différentes phases de jeux et des variations de rythme qui vont se répercuter sur les constructions linguistiques et prosodiques. Cette observation est sans doute encore plus visible si on compare un match d'un sport collectif avec une performance sportive individuelle, ou encore une course.

L'idée est de montrer in fine que le commentaire sportif en direct est entièrement influencé par ses conditions de production et qu'on peut expliquer les structures linguistiques caractéristiques de ce genre par l'analyse fine de ces conditions.

\section{Caractérisation du genre de discours et contraintes}

Richer (20II) propose de regrouper les différents aspects définitoires du genre selon cinq dimensions :

- la dimension matérielle comprenant les caractéristiques formelles du médium ainsi que la dimension spatiale et/ou temporelle;

- la dimension socio-pragmatique qui regroupe la détermination des coénonciateurs, l'identification du système d'énonciation global, l'identification de l'acte de parole et les dimensions du logos, de l'ethos et du pathos;

- la dimension textuelle, c'est-à-dire l'ensemble des éléments portant sur l'organisation du texte en séquences;

- la dimension stylistique, c'est-à-dire les structures linguistiques privilégiées dans le genre;

- la dimension thématique, c'est-à-dire le contenu thématique privilégié pour un genre donné.

Un rapide état des lieux de la littérature sur les genres de discours (voir, par exemple, Charaudeau, 2002), montre qu'une bonne partie des approches définitoires des genres, qu'elles soient liées aux pratiques sociales, aux fonctions de l'acte de communication, aux marques énonciatives ou à la nature communicationnelle de l'interaction, portent essentiellement sur la dimension socio-pragmatique. Ainsi, 
pour le genre de discours qui nous intéresse ici, à savoir le commentaire sportif télévisé, Deulofeu (2000) insiste sur la nécessité d'étudier le genre non seulement selon un point de vue interne au discours, qui serait celui des structures linguistiques, mais aussi en prenant en compte un point de vue externe au discours, qui englobe la spécificité pragmatique. Pršir et al. (2013) utilisent de la même manière des traits de description pragmatique - caractère médiatique, type d'audience, degré de préparation et degré d'interactivité - pour caractériser et regrouper des genres de discours oraux.

Cette caractérisation du genre selon la dimension socio-pragmatique amène pour certains genres de discours, et particulièrement pour le commentaire sportif en direct, à observer une grande diversité des styles, dépendant notamment du sport commenté (Audrit et al., 20I2), du journaliste sportif, de la nature du médium (commentaire radiophonique ou télévisuel)... Si on se réfère à nouveau aux dimensions proposées par Richer (20II) qui définissent le cadre générique, on se rend compte que l'hétérogénéité du genre du commentaire sportif dépend essentiellement de la dimension matérielle, à savoir la nature du médium et la nature du sport commenté. Ainsi, caractériser le genre en n'utilisant que la dimension socio-pragmatique nous amène soit à construire une arborescence complexe des genres en sous-genres, soit à accepter qu'au sein d'un seul et même genre de discours coexistent des collections de styles. On en arrive à la conclusion que la seule dimension socio-pragmatique n'est pas un outil de description suffisant pour expliquer les productions linguistiques propres au genre. Comme Krazem (20II), nous pensons que la dimension matérielle doit être prise en compte, non pas tant pour faire des sous-classifications (générèmes ${ }^{1}$ ) à l'intérieur du genre du commentaire sportif, que pour déterminer les contraintes qui vont influencer directement les productions linguistiques. Ainsi, pour expliquer les productions linguistiques spécifiques au genre du commentaire sportif, il nous semble nécessaire de les examiner en tenant compte:

- des contraintes de genre qui regroupent la dimension socio-pragmatique (caractère médiatique, rôle respectif des co-énonciateurs, construction faussement dialogale...) ainsi que la dimension thématique (le commentaire sportif porte essentiellement sur le sport commenté, les thèmes peuvent légèrement varier selon les sports...);

- des contraintes matérielles qui regroupent la nature du médium (radio ou télévisuel) mais également la dimension temporelle, ainsi que la nature et le rythme du sport commenté.

Prenons par exemple la répartition de la parole dans le commentaire sportif, d'une part cette répartition est contrainte par les rôles respectifs du journaliste sportif et de l'expert, ces rôles des co-énonciateurs sont définis par des contraintes

1. Générèmes: «figures génériques minimales insécables» présentées dans le cadre d’une structure de traits. 
de genre. Mais par ailleurs, les temps de parole respectifs sont répartis en fonction de la nature des événements commentés et du rythme du sport, c'est-à-dire des contraintes matérielles.

Ainsi, si l'objectif ultime de notre étude est de décrire les structures linguistiques (lexicales, syntaxiques) ainsi que les phonostyles (réalisations prosodiques de ces structures linguistiques) propres à un genre de discours, il nous semble que la définition d'un «cadre générique», tel qu'il est proposé traditionnellement dans la littérature, ne permet pas d'atteindre ce niveau de description. En effet, de par sa définition même, le cadre générique (Richer, 20II, citant Canvat, 1999) couvre le niveau discursif. C'est pourquoi, nous proposons de définir plutôt un set de contraintes qui englobe notamment la dimension matérielle, celle-ci influençant directement le niveau phrastique. On généralise ensuite ce système de contraintes à l'ensemble des autres dimensions, et notamment les dimensions pragmatique et thématique.

Ainsi, reprenant les six dimensions de Richer (20II) dont la dimension culturelle qui englobe les cinq autres, nous pouvons schématiser dans la figure I les relations qui sont établies entre ces différentes dimensions et les sets de contraintes que nous avons identifiés.

- Les contraintes de genre sont centrales; elles sont reliées à toutes les dimensions. Elles ont un impact sur la sélection thématique : le commentaire sportif commente essentiellement du sport. Ce sont les contraintes du genre qui imposent la présence d'au moins deux co-énonciateurs, un journaliste et un expert-consultant, elles jouent donc sur la dimension socio-pragmatique. Elles sont reliées à la dimension matérielle également: la nature du médium (diffusé médiatiquement, via la radio, la presse écrite ou la télévision) influe sur certains aspects de ce genre de discours, comme par exemple la nécessité de capter l'attention du public... Et bien sûr, les contraintes de genre influent en partie sur l'organisation textuelle et sur les productions stylistiques. Ce sont, par exemple, les contraintes de genre qui obligent le journaliste à produire des structures qui suggèrent une «émotion du sport».

- Reliées à la dimension matérielle d'une part et à la dimension thématique d'autre part, nous avons fait émerger des contraintes de média et des contraintes de contenu. Les contraintes du média sont liées à la taille ou à la temporalité du discours, à sa dimension orale et visuelle (ce qui a un impact sur la sélection de l'information par exemple).

- Les contraintes de contenu recouvrent le fait que le contenu du discours est imposé au locuteur par ce qui se passe sous ses yeux. Cela a un impact également sur le rythme de production du discours par exemple. Les contraintes de contenu ne sont généralement pas prises en compte comme éléments définitoires d'un genre de discours, au-delà de la dimension purement thématique. Pourtant, dans le cas du commentaire sportif en 
direct, ces contraintes de contenu deviennent excessivement importantes pour expliquer la forme du discours, dans la mesure où le commentaire dépend de ce qui est présenté sous les yeux du commentateur, et qu'ainsi le contenu du commentaire et son rythme sont dictés au commentateur par l'action qui se déroule sur le terrain. Par exemple, le journaliste ne peut pas mentionner une phase de jeu qui ne serait pas liée directement avec la phase en cours sur le terrain. Il ne peut le faire que dans les «temps morts» et dès que l'action sur le terrain reprend, le journaliste mentionne uniquement les actions en cours.

Ces trois sets de contraintes - de genre, de contenu et de média - ont un impact direct sur la dimension textuelle et sur le choix des structures et du lexique. En considérant le commentaire sportif comme un discours contraint, non seulement par le genre, mais aussi par les dimensions de médium et de contenu, notre démarche est d'analyser l'influence de ces contraintes sur le discours des journalistes et ainsi de non seulement relever des structures propres au genre, mais surtout d'expliquer la raison de ces structures et leurs variations à l'intérieur du genre.

\section{Organisation du contenu et influence sur le discours}

Comme nous l'avons mentionné plus haut, le commentaire sportif en direct présente cette particularité qu'il est fortement influencé par le contenu qu'il commente. Cette contrainte sur le discours amenée par le contenu doit être prise en compte et nécessite de fait une analyse du contenu en lui-même. Il faut être en mesure de comprendre l'organisation du contenu pour comprendre son influence sur le commentaire. Nous prenons ici l'exemple d'un match de rugby, mais une méthodologie identique peut être appliquée à tous les événements médiatiques qui font l'objet d'un commentaire télévisuel en direct.

Le match de rugby est composé de phases de jeu: phases statiques comme les touches par exemple, phases de fixations comme le maul et phases de plein mouvement comme par exemple la contre-attaque (pour le détail des phases de jeu, voir notamment Mouchet et al., 2005). Ces phases de jeu sont composées de différents événements, qui peuvent eux-mêmes être décomposés en plusieurs actions. La figure 2 montre la composition du jeu en phases, événements et actions.

Si le contenu a un impact sur le commentaire c'est sur la base d'une contrainte générale de fidélité entre l'input (le jeu) et l'output (le commentaire). Cette contrainte de fidélité est propre au genre du commentaire en direct. Elle s'applique sur les événements marquants, «Fid(EvM)» au sein des phases de jeu, et sur les actions proéminentes au sein des événements, «Fid(ActP)». Ainsi, parmi les différentes phases de jeu, certains événements doivent être absolument mentionnés parce qu'ils participent à la compréhension de la dynamique et des enjeux du match. À l'intérieur des événements, certaines actions plus représentatives, ou ayant un impact sur le jeu dans sa dimension globale, doivent également être mentionnées. 


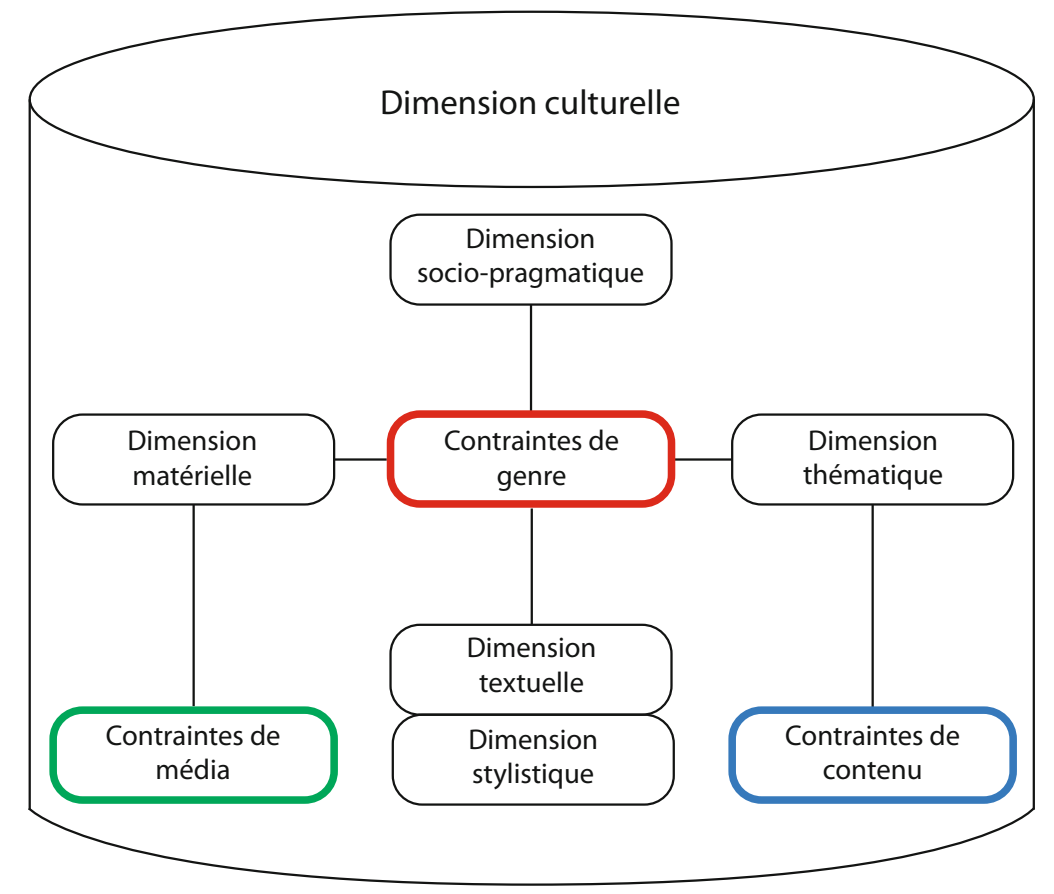

Figure 1. Schéma des relations entre dimensions définitoires du genre et contraintes de genre, de média et de contenu

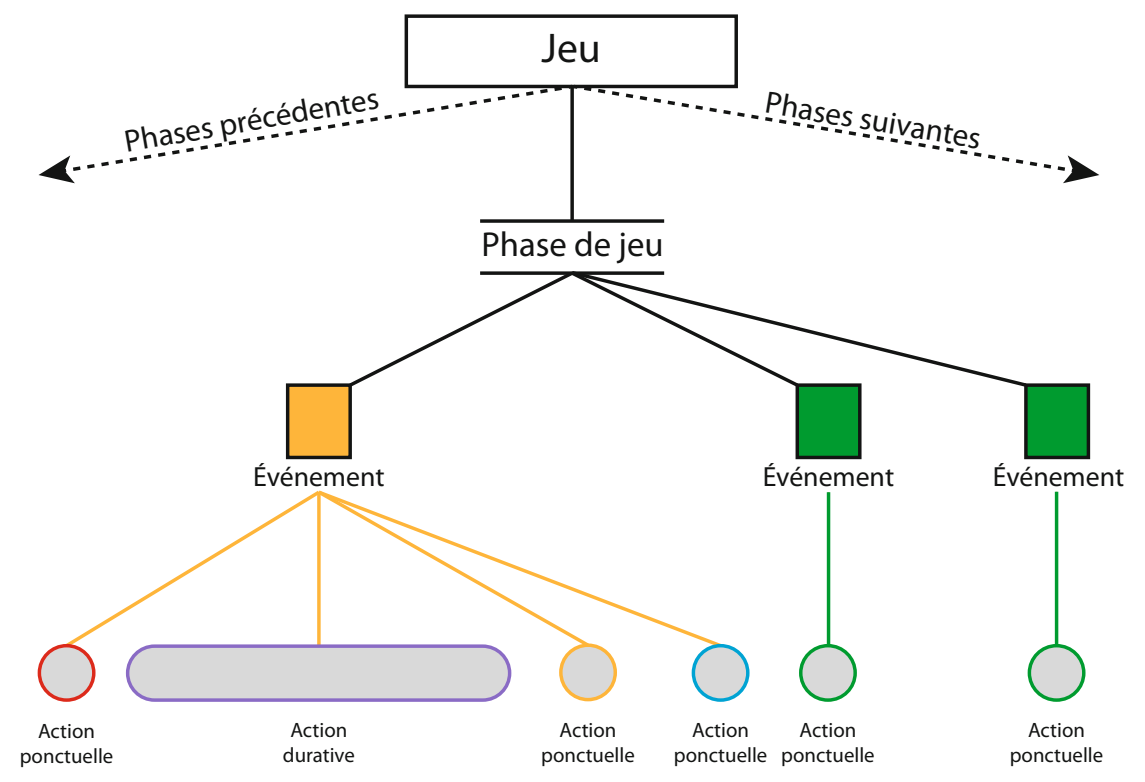

Figure 2. Composition du jeu d'un match de rugby 


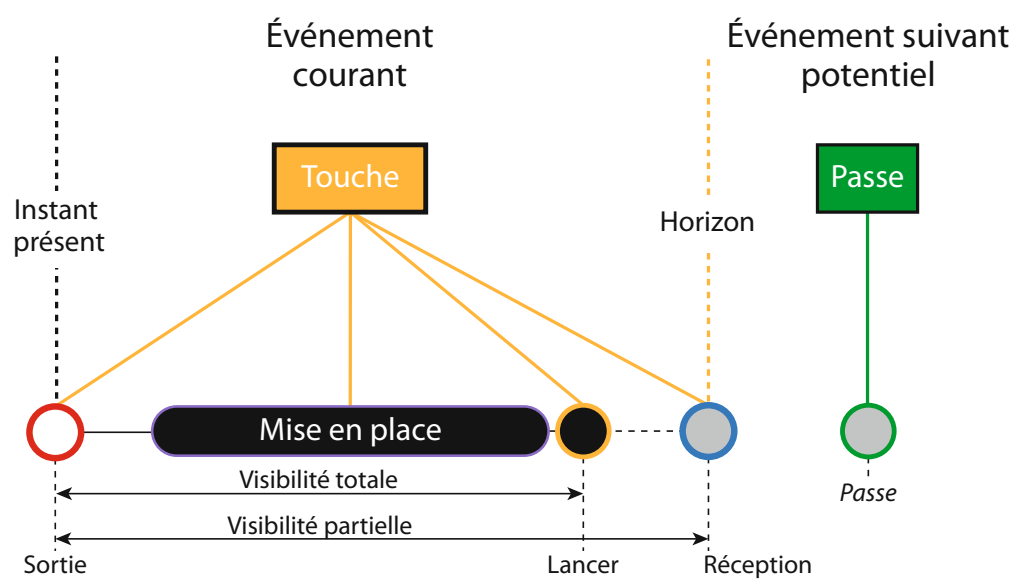

Figure 3. Déroulement de l'événement «touche» dans un match de rugby

Nous allons donner l'exemple d'une phase statique, la touche. La touche est un événement marquant. Elle est systématiquement mentionnée. Elle se compose de quatre actions successives:

- la sortie: action ponctuelle et proéminente,

- la mise en ligne: action durative et secondaire,

- le lancer: action ponctuelle et secondaire,

- la récupération: action ponctuelle et proéminente.

Sur les quatre actions qui composent l'événement «touche», seules les actions proéminentes, sortie et récupération sont mentionnées à chaque fois. Les actions secondaires, la mise en ligne et le lancer, ne sont presque jamais indiquées.

La figure 3 représente le déroulement de l'événement «touche». Les actions ponctuelles sont représentées par des points de différentes couleurs en fonction du type de l'action, les actions duratives par des rectangles. Les actions proéminentes sont représentées par un contour plus épais que les autres actions. Ainsi, l'action «sortie» est représentée avec un contour plus épais (en rouge) que le contour de l'action «mise en place» (en violet).

Les noyaux des cercles qui représentent les actions indiquent l'équipe en possession du ballon : noir : équipe $A$; blanc: équipe $B$; gris : équipe $A$ ou équipe $B$ (incertitude). La visibilité représente le temps disponible estimé avant la prochaine action (à l'intérieur de l'événement en cours). La mise en ligne (des joueurs) permet une visibilité maximale, parce que c'est une action durative avec une manifestation visuelle du progrès de l'action et de son achèvement. La visibilité partielle est due à la connaissance de la structure de l'événement et concerne une certaine incertitude par rapport au rythme exact d'enchaînement des actions. 
Le contenu influence également le discours en différé, c'est-à-dire le discours produit de manière asynchrone par rapport à la réalisation des actions commentées: c'est le cas des actions qui font l'objet d'un replay à vitesse normale ou d'un replay au ralenti. Si le contenu contraint également le commentaire, au sens où celui-ci doit porter sur l'action qui est rejouée, il contraint moins, en revanche, le locuteur quant au choix des structures linguistiques. En effet, sur ces séquences de replay, le commentateur a déjà une connaissance de l'action à commenter, il a un temps de préparation suffisant pour organiser son texte et choisir ses structures. En direct, la synchronisation en temps réel du commentaire avec les actions est plus contraignante pour le commentateur. On notera que c'est souvent le consultant qui produit les commentaires sur les replays et ralentis, tandis que le journaliste est en général chargé de produire les séquences en direct.

\section{Formalisation des contraintes et hiérarchisation}

Nous présentons dans cette section le détail des contraintes que nous avons identifiées en rapport avec la production du commentaire sportif télévisé en direct. Afin de formaliser l'analyse des contraintes extralinguistiques sur le discours et de proposer une hiérarchisation de ces contraintes, nous utilisons l'appareillage de la théorie de l'optimalité (Prince et Smolensky, 1993) que nous appliquons au domaine discursif.

La figure 4 représente les trois blocs de contraintes, ou plutôt les trois dimensions qui contraignent le discours, et leurs relations avec l'organisation et la construction du discours. Le schéma est ainsi organisé en trois grandes parties:

- un premier bloc (en haut, à gauche) représente les contraintes de genre, qui recouvrent principalement les dimensions socio-pragmatique et thématique;

- le second bloc, au centre, représente les circonstances matérielles de production du discours (voir la dimension matérielle dans Richer, 20II);

- le dernier bloc (en haut, à droite) représente le contenu qui fait l'objet du commentaire.

Ces trois groupes de contraintes influent ensemble sur le commentaire produit et sur la répartition de la parole entre les deux co-énonciateurs - le journaliste et le consultant -, ceux-ci faisant appel à la fois aux ressources (compétences) linguistiques à leur disposition et à leur connaissance encyclopédique du domaine.

Ces groupes de contraintes sont liés les uns aux autres au sens où, d'une part, les trois agissent ensemble sur l'organisation textuelle, la répartition de la parole entre le journaliste et le consultant, et bien sûr le choix des structures linguistiques adaptées; et, d'autre part, le lien entre les trois blocs de contraintes provient aussi de ce que certaines composantes de ces contraintes dépendent d'au moins deux des blocs. Prenons, par exemple, la nature télévisuelle et en direct du commentaire. Celle-ci est prise en compte dans les contraintes de genre, dans la mesure où nous pensons que 


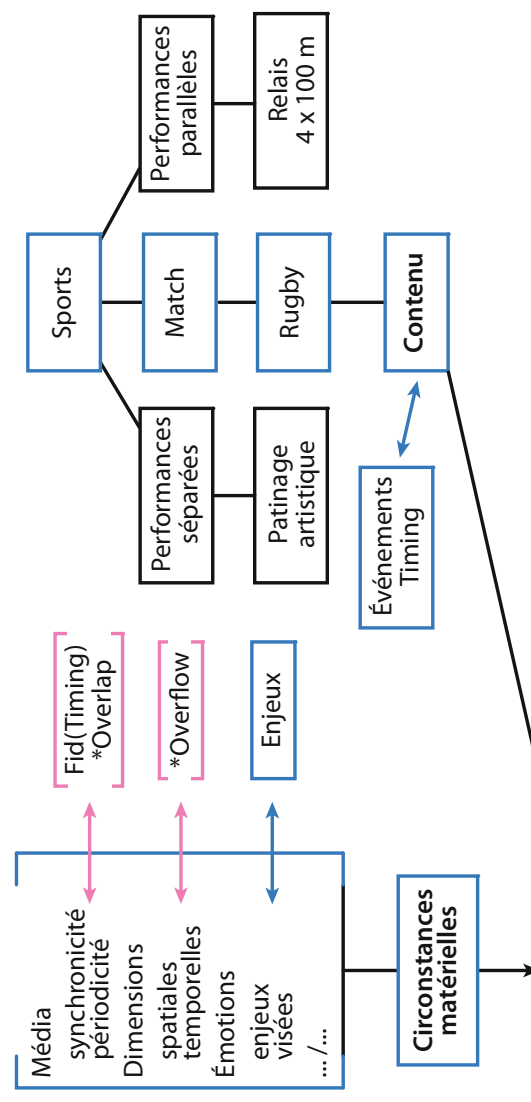

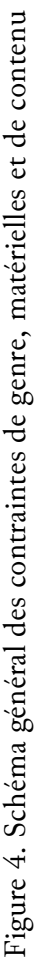


le commentaire sportif télévisuel en direct est effectivement un genre de discours à part entière qui va influer par exemple sur le choix du nombre de co-énonciateurs construisant le commentaire et sur leurs rôles: le genre du commentaire sportif en direct attend en général deux co-énonciateurs, un journaliste sportif et un consultant, ancien sportif et spécialiste du sport commenté. Mais la nature télévisuelle et en direct du commentaire sportif est également une composante des circonstances matérielles, qui joue par exemple sur la nécessité de synchroniser une partie du commentaire avec les images diffusées par la régie aux téléspectateurs. L'interaction entre les contraintes dites de genre et les deux autres types de contraintes tient notamment au phénomène d'institutionnalisation que nous décrivons en section 7 .

\subsection{Contraintes du genre de discours}

Le premier bloc, en haut à gauche de la figure 4, représente les contraintes de genre. Le genre de discours est représenté comme une ontologie au sein de laquelle le commentaire sportif en direct n'est en fait qu'un sous-genre du commentaire télévisé en direct. Sous le commentaire télévisé en direct, on trouve non seulement le commentaire sportif, mais également le commentaire d'autres événements médiatiques tels que les parades militaires, les mariages princiers, les soirées électorales... Par ailleurs, le commentaire télévisé en direct appartient lui-même à une catégorie plus générale, celle du discours médiatique.

À différents niveaux de cette ontologie du discours médiatique viennent s'appliquer des contraintes. Au niveau supérieur, c'est-à-dire au niveau du discours médiatique, on applique une contrainte «Max(Émo)» qui vise à transmettre un maximum d'émotions à travers le commentaire. En effet, le commentaire sportif en tant que genre médiatique est destiné à un public de téléspectateurs qui doit rester captivé, pour assurer les audiences de la chaîne de diffusion. Ainsi, le journaliste doit s'assurer que le match et le commentaire constituent un spectacle digne d'intérêt. Une des clefs du spectacle, c'est de fournir une émotion, celle ressentie dans le stade et transmise par le commentateur.

Au niveau du commentaire en direct, la contrainte «Fid(Événement)» est appliquée. Elle demande que le commentaire en direct soit en correspondance avec le contenu commenté, et ce quels que soient le contenu et le médium. Pour un commentaire sportif en direct d'un match de rugby, dont le contenu est divisé en phases de jeu, elles-mêmes subdivisées en événements et en actions, la contrainte «Fid(Événement)» implique qu'une partie du commentaire soit consacrée à décrire les événements du match. Cette contrainte s'applique que le médium soit la radio, la télévision ou encore un flux internet (minutes du jeu). Le commentaire ne peut pas couvrir l'ensemble des actions du match, les locuteurs sont amenés à sélectionner les événements marquants et les actions proéminentes du jeu. La contrainte «Fid(Événement)» peut être détaillée ainsi : «Fid(EvM)» implique une description des événements marquants du jeu, c'est-à-dire les événements qui peuvent impacter le score des deux équipes; «Fid(ActP)» contraint le locuteur à mentionner les actions les plus visibles et/ou les plus importantes d'un événement ou d'une phase de jeu. 
La contrainte «Max(Comp)» est une contrainte spécifique au commentaire sportif. Elle demande que le commentateur fournisse un maximum d'informations complémentaires plutôt que des descriptions de ce qui se passe sur le terrain, descriptions qui, vu le caractère télévisuel du genre, pourraient s'avérer redondantes. L'apport du commentaire sportif repose en partie en effet sur les informations et connaissances tactiques et stratégiques que le journaliste et le consultant ont du jeu, et qui permettent au téléspectateur de mieux saisir la dynamique et les enjeux du match. L'annotation du commentaire sportif en périodes descriptives et périodes complémentaires (Augendre et Mathon, 20Iza et b) permet de déterminer la part du commentaire complémentaire: $52,5 \%$ des tours de parole sont destinés à offrir des informations complémentaires, contre presque $44 \%$ de tours de parole centrés sur la description du jeu.

La contrainte «Max(Comp)» a bien sûr un lien avec le type de média. Elle peut ainsi d'autant mieux être remplie que le téléspectateur bénéficie d'un visuel sur le match, et que la description des actions est moins cruciale pour la compréhension du jeu. Ainsi, les contraintes que nous définissons indépendamment au sein de trois grandes classes, contraintes de contenu, contraintes de média et contraintes de genre, interagissent pour autant les unes avec les autres. Au contraire, nous cherchons à montrer que seule la prise en compte de ces trois dimensions ensemble permet de réellement rendre compte de la réalité du fonctionnement de ce genre de discours.

\subsection{Contraintes liées aux circonstances matérielles}

Les contraintes liées aux circonstances matérielles sont notées dans le bloc supérieur central de la figure 4. Les circonstances matérielles sont directement issues de la dimension matérielle telle que définie par Richer (20II). Ces circonstances n'ont pas forcément de relations les unes avec les autres et sont présentées de ce fait comme une liste de traits, et non pas comme un ensemble hiérarchisé (ce qui était le cas des contraintes de genre). Une présentation possible de ces circonstances matérielles serait l'utilisation de traits tels que ceux utilisés par Krazem (20iI) pour construire des «générèmes». Nous avons choisi d'établir ici simplement une liste des circonstances matérielles (basée sur Richer, 20II) pertinentes par rapport au commentaire sportif en direct.

Nous avons identifié deux contraintes liées à la nature audiovisuelle du médium.

- La contrainte «Fid(Timing)» implique une synchronisation du commentaire avec les images des actions de jeu, images sélectionnées et diffusées par la régie.

- La contrainte «*Simult» découle directement de la nature du médium, qui demande que la gestion de la prise de parole ne soit pas celle que l'on observe dans une conversation naturelle. En effet, pour la bonne compréhension du commentaire par les téléspectateurs, les locuteurs doivent éviter au maximum de parler de manière simultanée. Cette contrainte «*Simult» contraste avec la mise en scène dialogique du commentaire sportif, contrainte de genre, 
qui impose la présence de deux locuteurs, un journaliste et un consultant. Si la mise en scène suggère une situation de parole qui ressemble à une conversation, les propriétés textuelles du commentaire réfutent complètement cette hypothèse. En effet, la parole simultanée ne représente que 2,6 \% du temps de parole, ce qui est bien inférieur au temps de parole simultanée attendu dans une conversation naturelle, où les locuteurs parlent simultanément environ $60 \%$ du temps de parole (Campbell, 2008). La contrainte «*Simult» qui influe pour permettre l'intelligibilité du discours médiatique oral, s'impose sur la mise en scène de la situation de parole.

Au niveau de la dimension temporelle du match s'applique la contrainte «*Dépas»: le commentaire ne doit pas dépasser la durée de l'action commentée. Cette contrainte est liée bien sûr à la contrainte de synchronisation du commentaire avec l'action, «Fid(Timing)», ainsi qu'aux spécificités du contenu, et notamment à la fenêtre de visibilité dont le locuteur dispose pour placer son commentaire (voir section 3 et figure 3 ).

Nous avons ajouté aux circonstances matérielles une dimension supplémentaire, celle des enjeux de l'événement et des émotions qui en découlent. L'événement médiatique commenté (sport ou autre) revêt des enjeux plus ou moins importants, qui influent sur l'apport d'émotions (qui constitue par ailleurs une contrainte du genre médiatique). Prenons l'exemple du match de rugby France-Argentine du 7 septembre 2007: c'est un match de Coupe du monde, il débute le championnat, l'une des deux équipes est celle du pays (la France) qui héberge la Coupe du monde. Le match est commenté en français et diffusé sur $\mathrm{TF}_{\mathrm{I}}$, la plus importante des chaines de diffusion privées et dont l'accès est gratuit pour les téléspectateurs. Ce match est un match important à tous points de vue: les enjeux du match vont influer sur la chaîne de diffusion, le choix du journaliste pour commenter le match, les spécificités du public (pas forcément spécialiste de rugby), ainsi que les attentes en termes de spectacle des téléspectateurs. L'ensemble de ces éléments constitue également des contraintes qui pèsent sur le commentaire et influencent les productions linguistiques.

\subsection{Influence de l'événement sportif sur le discours}

Nous avons identifié un dernier ensemble influençant directement la production du commentaire en direct (en haut à droite de la figure 4): il s'agit du contenu. Nous entendons par contenu la nature de l'événement commenté, ici un événement sportif, par opposition à d'autres événements médiatiques tels que ceux que nous avons mentionnés ci-dessus: mariage princier, élections, etc. L'événement sportif peut être subdivisé en différentes catégories en fonction de la nature du sport: match, performance parallèle, performance individuelle, à dimension artistique ou non... Sous la catégorie match, on distinguera par ailleurs le match de rugby du match de football ou encore du match de basket. Le niveau de granularité des distinctions faites entre les sports est justifié par les observations d'Audrit et al. (20I2) concernant les différences de cinétique de ces sports (rugby, football et basket), et 
leur influence sur l'homogénéité des réalisations prosodiques. Ce sont les contraintes de genre «Fid(Événement)» et les contraintes de média «Fid(Timing)» notamment qui impliquent une telle importance de la nature - et notamment du rythme - du contenu par rapport au commentaire. Ainsi, nous n'avons pas ajouté de contraintes supplémentaires liées au contenu, étant entendu que les contraintes citées plus haut impliquent quoi qu'il en soit une relation forte et nécessaire entre le commentaire et le contenu commenté.

\section{Choix des annotations et outils pour la description}

Afin de concrétiser notre réflexion sur le commentaire sportif en direct et sur les différentes contraintes afférentes à ce type de discours, nous avons réalisé un ensemble d'annotations et d'analyses sur le commentaire sportif d'un match de rugby. L'observation du discours transcrit a constitué notre point de départ, dans le prolongement des différentes études déjà publiées sur ce type de discours (Deulofeu, 2000; Hartmann, 20II). Cependant, la nécessité de considérer et d'évaluer le poids des contraintes extralinguistiques sur le discours est assez rapidement devenue notre principale préoccupation, faisant ainsi la spécificité de notre recherche.

Le commentaire sportif «test» sur lequel nous avons réalisé nos analyses jusqu’à présent est le commentaire télévisé d'un match de rugby de la Coupe du monde 2007, opposant la France et l'Argentine ${ }^{2}$. Si la sélection d'un match (ses équipes, ses enjeux, ses commentateurs...) et d'un sport (rugby vs tennis vs patinage artistique...) restreint de fait la portée des résultats à une catégorie de commentaire sportif, les annotations et analyses réalisées sont, quant à elles, applicables à tout commentaire sportif: les contraintes restent globalement les mêmes, et seuls leur poids respectif (hiérarchisation des contraintes) et leur niveau de visibilité dans le discours par rapport à un sport spécifique (lexique, prosodie, syntaxe...) varient.

À partir des données disponibles au départ, c'est-à-dire la transcription du commentaire de ce match réalisée avec Transcriber et alignée sur le fichier son avec WinPitch 7 (Lortal et Mathon, 2008), nous avons procédé à différentes annotations, à la fois sur le discours et sur la vidéo de l'événement sportif.

\subsection{Périodes descriptives / périodes explicatives}

Dans le prolongement du travail de Deulofeu (2000) sur le commentaire sportif, la première étape de notre analyse portait sur la structure textuelle et consistait à distinguer, grâce à des balises «section» dans l'interface Transcriber, deux soustypes de productions: celles qui relèvent de la description et celles qui relèvent de l'explication. En parlant de description et d'explication, nous faisons référence aux

2. Le match commenté est d'une durée de i heure et 46 minutes environ, pour I6 I5o mots. Il est composé de 2050 tours de parole, c'est-à-dire des segments de parole séparés les uns des autres par des pauses d'au moins $200 \mathrm{~ms}$, et/ou une alternance du locuteur en charge du tour de parole. 
actes de parole qui conduisent ces périodes descriptives et explicatives. En termes de structure textuelle, les périodes descriptives se rapprocheraient plutôt d'une structure narrative (exemple de la chaîne référentielle, voir Augendre et Mathon, zoIza et b).

Par discours descriptif, nous entendons le discours produit pour décrire ce que les commentateurs et téléspectateurs voient sur l'écran, ce que Hartmann (20II : 30) désigne comme du «play-by-play». Ce type de discours a pour principale caractéristique d'être produit de façon synchrone à l'événement sportif: c'est sur lui qu'agissent principalement les contraintes de genre, «Fid(Événement)», et de média, «Fid(Timing)».

Par discours explicatif, désigné comme du «color-commentary» par Hartmann (20II : 30), nous entendons le discours annexe, qui ne décrit pas ce qui est visible mais fournit des informations supplémentaires, sans lien direct avec l'événement sportif en cours. Ce type de discours résulte de la contrainte de genre, «Max(Comp)», attachée au niveau du commentaire sportif. La présence de ce type de discours est largement dépendante du jeu, au sens où elle est favorisée à certains moments du match, quand les images montrent des événements suffisamment longs pour permettre des interventions moins contraintes du point de vue de la structure, avec une bonne visibilité par rapport au temps de parole alloué avant la prochaine action proéminente à mentionner.

La distinction discours descriptif/discours explicatif constitue une première étape, nécessaire mais pas suffisante, pour produire une analyse pertinente du commentaire sportif: en effet, elle ne considère que la structure textuelle et ne rend pas compte des contraintes qui affectent directement et l'organisation textuelle (notamment la répartition de la parole) et les productions stylistiques.

\subsection{Annotation syntaxique}

En parallèle d'une annotation de l'événement sportif, nous avons poursuivi l'analyse du discours en commençant par le niveau syntaxique. L'annotation des structures syntaxiques du corpus, réalisée manuellement, a été effectuée dans le seul but d'être confrontée à celle de l'événement sportif et en aucun cas à des fins purement descriptives: l'objectif n'est pas de décrire la syntaxe du commentaire mais d'envisager la syntaxe comme un des niveaux où les contraintes extralinguistiques sont susceptibles de se manifester.

À partir de la liste de structures proposée par Deulofeu (2000) dans une étude sur le commentaire d'un match de football, nous avons annoté les tours de parole de notre corpus (correspondant à des groupes de souffle) en utilisant les types suivants:

- phrase simple,

- phrase complexe,

- groupe nominal (en distinguant les noms avec et sans article),

- nom propre,

- nom propre + relative en «qui» (exemple: «Mignoni encore qui insiste au ras»), 
- préposition + nom propre ("pour Skrela»),

- groupe prépositionnel («avec le départ de Roncero»),

- forme verbale non finie (infinitif/participe),

- interjections.

Au-delà de ces catégories, nous avons, entre autres, distingué des degrés de complexité pour chaque structure (GP simple vs GP + GP; nom sans article seul vs $\mathrm{N}+\mathrm{GP}$ ) et listé les noms communs utilisés sans article ainsi que les prépositions présentes dans les groupes prépositionnels produits comme des segments autonomes.

Sur la base de leur fréquence dans notre corpus, nous avons retenu les cinq structures suivantes comme «spécifiques» du "play-by-play»: les structures autour d'un nom propre, avec (prép $+\mathrm{NP}$ ) ou sans préposition (NP), les structures autour d'un nom, avec (GN) ou sans article (N) et enfin les groupes prépositionnels (GP). Les phrases verbales, bien que globalement majoritaires sur l'ensemble du commentaire, sont délaissées dans le "play-by-play» au profit de structures moins coûteuses, plus courtes mais syntaxiquement ouvertes, mettant au jour l'influence des contraintes de fidélité à l'action et de timing sur le discours. L'introduction d'un agent par le biais d'un nom propre seul, par exemple, peut suffire à constituer un tour de parole ou peut, en fonction du degré de visibilité, être complétée par une relative en "qui» permettant, dans un deuxième temps, d'introduire l'action associée à l'agent qui vient d'être introduit. La fréquence de ces structures spécifiques, construites autour de noms et permettant à la fois de stopper ou de poursuivre le discours, constitue une des manifestations des contraintes «Fid(Timing)» et «*Dépas».

\subsection{Annotation de l'événement sportif: actions et événements de jeu}

Afin de rendre compte du fait que le commentaire sportif est un discours produit sous contraintes, nous avons dépassé les limites du discours et choisi d'annoter l'événement sportif, indépendamment du commentaire qui lui est associé. Pour ce faire, nous avons annoté l'ensemble des actions et événements du match. L'annotation a été réalisée manuellement puis intégrée à la vidéo, sous la forme de sous-titres, grâce au logiciel Aegisub ${ }^{3}$.

L'annotation des actions et phases de jeu avait pour but de rendre compte du rythme du jeu. Pour pouvoir évaluer le rythme du jeu et le corréler avec le rythme du discours, nous avons défini trois plages de rythme, sur la base du nombre d'actions réalisées dans un laps de temps de 5 secondes. La plage I représente le rythme le plus lent, de i à 3 actions réalisées en 5 secondes; la plage 2 correspond à un rythme moyen, de 4 à 6 actions réalisées en 5 secondes; la plage 3 représente le rythme le plus rapide, de 7 à Io actions réalisées en 5 secondes.

Par ailleurs, nous avons distingué les actions commentées des actions non commentées et associé à chaque action commentée la description qui lui correspond.

3. Voir: http://www.aegisub.org/. 


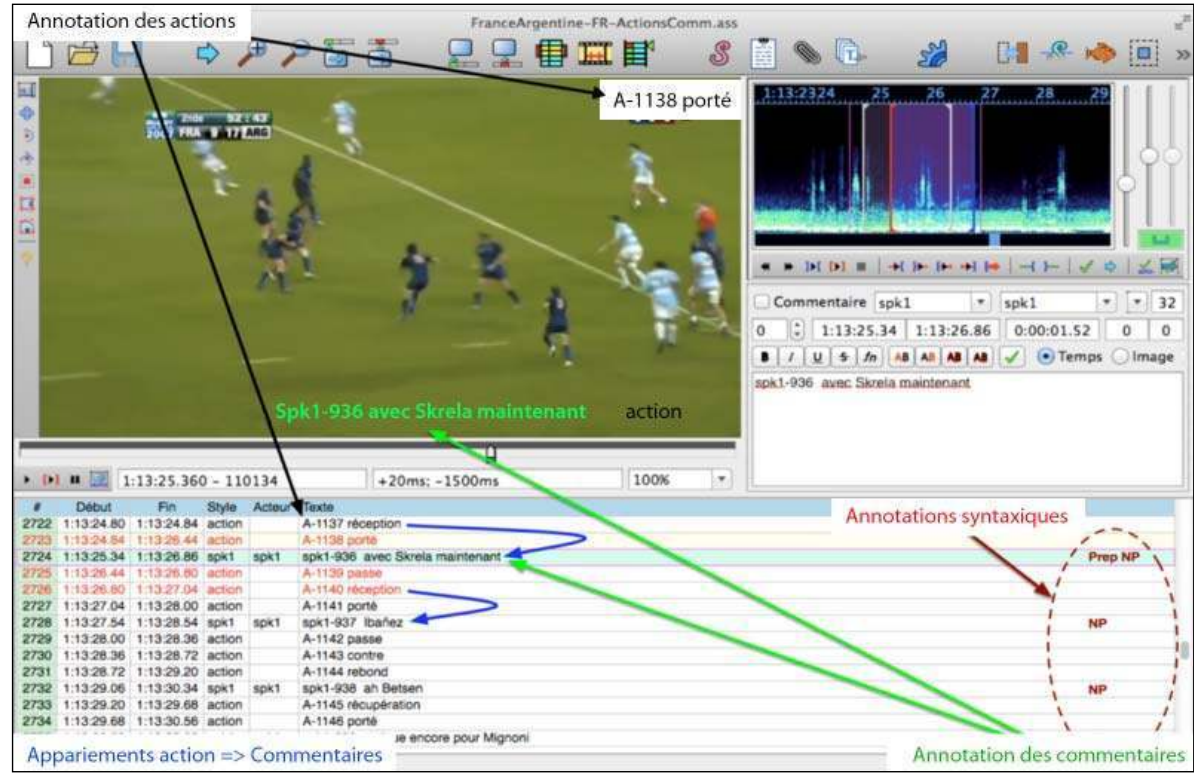

Figure 5. Capture d'écran des annotations de jeu et de discours affichées avec l'interface Aegisub

\subsection{Représentation des données et exemples de croisements}

Grâce à l'ensemble des annotations que nous avons réalisées, le fichier vidéo du match nous donne à présent accès à un grand nombre d'informations simultanées facilitant l'analyse:

- image,

- son,

- données temporelles relatives au match (chronomètre) et aux actions (début-fin),

- nom des actions,

- locuteur,

- texte des commentaires aligné sur le son et apparié à l'action,

- annotation syntaxique.

La capture d'écran ci-dessus (figure 5 ) permet de rendre compte des informations accessibles.

La mise en commun des annotations que nous avons effectuées en parallèle sur la vidéo et sur le discours nous permet donc de voir en temps réel quelles actions sont commentées, quand, par qui et comment. Grâce à ces croisements, nous avons accès, entre autres:

- aux informations relatives aux actions commentées et non commentées corrélées au locuteur et/ou à la syntaxe des tours de parole; 
- aux informations relatives à la distribution de la parole entre locuteurs et à la syntaxe des tours de parole pour chaque plage de rythme;

- aux informations relatives aux interventions des locuteurs (quand? comment? pourquoi?);

- aux données syntaxiques, en fonction du type de discours («play-by-play» ou «color-commentary»), du locuteur, ou encore de la plage de rythme.

C'est grâce à ces différentes annotations, réalisées en amont sur l'intégralité d'un événement sportif, que nous pouvons maintenant tester nos trois sets de contraintes relevant du genre de discours, des circonstances matérielles et du contenu, afin d'évaluer leur poids respectif et la façon dont ces contraintes se manifestent au sein du discours produit.

\section{Quelques exemples de l'influence des contraintes sur les productions linguistiques}

À partir des contraintes identifiées dans la section 4 et des annotations réalisées sur notre corpus, nous proposons dans cette partie de revenir sur quelques-unes des contraintes décrites dans la section 4, en illustrant leur influence sur les productions linguistiques des locuteurs, mais aussi sur l'organisation du texte, la répartition de la parole, en résumé sur la construction du commentaire.

\subsection{Illustration des contraintes de genre sur le discours}

L'une des premières contraintes du genre du commentaire sportif en direct est la contrainte «Max(Émo)» qui demande que le commentaire transmette de l'émotion, qui va retenir le téléspectateur et augmenter l'audience. Sur la figure 4, cette contrainte est notée au niveau du discours médiatique, et non pas au niveau du commentaire sportif en direct. En effet, c'est le caractère médiatique du commentaire sportif qui impose la transmission de l'émotion. En comparant deux commentaires en direct de deux événements médiatiques différents, le commentaire d'un match de rugby et celui du défilé militaire du i4 juillet, on s'aperçoit que les deux transmettent de l'émotion, même si la nature de l'émotion n'est globalement pas la même et par conséquent, les réalisations linguistiques et prosodiques varient fortement.

La contrainte «Max(Émo)» se manifeste dans le commentaire sportif à travers certaines formes linguistiques, comme par exemple certaines onomatopées et constructions exclamatives. L'émotion qui transparaît n'est d'ailleurs pas toujours la forme d'excitation positive qui est rattachée le plus souvent au sport. Ainsi, par exemple,

[I] oh qu'il doit s'en vouloir David qu'il doit s'en vouloir

marque plutôt un sentiment de déception. 


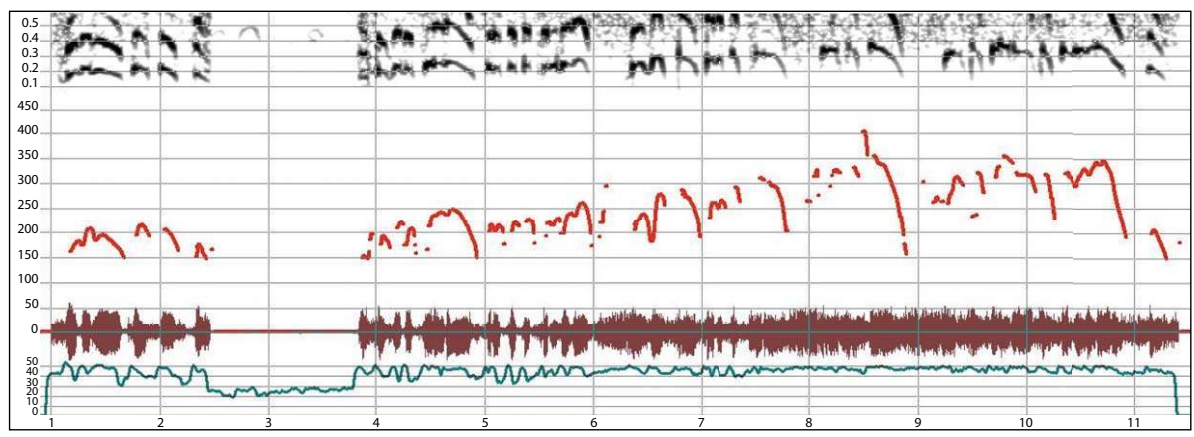

Figure 6. Représentation de la courbe mélodique (en rouge) de l'énoncé: «de Villiers qui insiste dans ce jeu de pilonnage qui va peut-être libérer des espaces avec Skrela qui est passé qui a passé les bras pour servir Heymans»

(figure extraite de Mathon et Boulakia [2009: 297]) ${ }^{4}$

Ce sont toutefois les réalisations prosodiques qui transmettent le mieux l'émotion du sport, notamment les montées de la fréquence fondamentale ( $\mathrm{Fo}$ ), sous forme de pics brusques ou en plateaux (augmentation progressive du registre de voix), accompagnées très souvent d'une augmentation du débit de parole.

La figure 6, extraite de Mathon et Boulakia (2009: 297), montre l'augmentation progressive de la Fo. On passe d'un registre moyen-bas pour le locuteur (entre 150 et $200 \mathrm{~Hz}$ ) à un registre haut, avec un pic de Fo à $400 \mathrm{~Hz}$.

On l'a dit, d'un événement médiatique à un autre, l'émotion transmise est différente et les productions linguistiques et prosodiques engendrées par la contrainte «Max(Émo)» varient également. C'est aussi le cas d'un sport à l'autre. En comparant un sport de type match avec un sport présentant une dimension artistique, comme le patinage sur glace, on s'aperçoit que l'émotion n'est pas transmise de la même manière. Ainsi, la musique est une dimension essentielle du patinage. Accompagnant les évolutions et les figures du sportif, elle est un vecteur d'émotion. Afin de respecter l'impact de la musique sur la perception de la performance, les commentateurs sont amenés à produire beaucoup moins de commentaires, laissant de larges plages à la musique. On note que, pour une performance de patinage artistique, programme court aux Jeux olympiques de Sotchi en 20I4, le temps de parole, tous locuteurs confondus, ne représente que $20 \%$ du temps de la performance, tandis que, pour le match de rugby (Coupe du monde 2007), le temps de parole total couvre un peu plus de $70 \%$ du temps du match. Les stratégies de construction de l'émotion ne semblent donc pas les mêmes d'un commentaire sportif à l'autre et semblent dépendre de la nature du sport.

4. La longueur de l'énoncé représenté par cette courbe mélodique ne permet pas une transcription orthographique directement en dessous de la courbe. 


\subsection{Illustration des contraintes \\ liées aux circonstances matérielles et au contenu}

Parmi les contraintes liées aux circonstances matérielles, et notamment à la nature du médium, nous avons noté une contrainte «Fid(Timing)» qui demande que le commentaire soit synchrone le plus possible avec le contenu commenté, en l'occurrence ici l'action sportive. En analysant le contenu d'un match de rugby (section 3), nous avons vu que celui-ci était composé de différentes phases de jeu, elles-mêmes subdivisées en événements de durée variable. L'enchaînement des phases de jeu, et à l'intérieur de ces phases des événements, constitue le scénario du jeu et cet enchaînement implique un rythme qui varie selon les phases. Si la contrainte «Fid(Timing)» s'exerce effectivement comme nous le pensons, alors la variation du rythme du jeu implique une variation du rythme dans le commentaire. Nous avons voulu analyser l'influence du rythme du jeu sur le rythme du commentaire en examinant notamment la répartition de la parole entre les deux commentateurs, en fonction du rythme du jeu. Partant du principe que les deux locuteurs ne possèdent pas les mêmes compétences linguistiques et ayant observé que le journaliste semblait intervenir dans les périodes de discours les plus contraintes, nous avons examiné la répartition de la parole entre les locuteurs qui construisent le commentaire (essentiellement le journaliste sportif et le consultant expert), et cela en fonction de trois phases de jeu. Nous avons étudié la répartition de la parole entre les deux locuteurs et par rapport à ces trois phases en faisant intervenir les contraintes que nous avons décrites plus haut.

Le tableau i ci-dessous décrit trois phases de jeu différentes (un essai, du jeu rapide, et un ralenti). Les colonnes correspondent aux contraintes ordonnées par importance décroissante (la contrainte la plus forte à gauche).

Dans le cas de l'essai, par exemple, le journaliste est le mieux placé pour prendre la parole et décrire l'événement. Ce n'est pas le moment pour l'expert de placer du discours complémentaire «(Comp)» puisqu'il s'agit d'un événement marquant, ce qui contreviendrait à la contrainte de fidélité «Fid(EvM)» - en l'occurrence, c'est cette infraction qui est décisive (*!). Dans cette situation, le journaliste pourrait même interrompre l'expert pour décrire l'essai puisque la contrainte de non-simultanéité, «*Simult», est inférieure à celle qui demande qu'on décrive les événements marquants, «Fid(EvM)». Dans le jeu rapide, ce sont les ressources linguistiques qui font la différence: en général, le journaliste dispose de structures atypiques qui lui permettent d'intervenir dans des fenêtres très courtes sans dépasser, tandis que l'expert serait amené à enfreindre la contrainte "*Dépas». Pour le ralenti, on a l'effet inverse, la contrainte la plus importante susceptible d'être enfreinte concerne le discours complémentaire «Max(Comp)» et favorise l'intervention de l'expert.

La figure 7 montre la répartition de la parole en nombre de tours de parole entre les différents locuteurs en fonction des trois plages de rythme (voir section 5.3). La première chose que l'on observe c'est que les deux locuteurs, que ce soit le journaliste ou le consultant/expert, privilégient la plage I, c'est-à-dire le rythme de jeu le plus lent, pour produire l'essentiel de leur commentaire. Ce qui différencie, en revanche, 


\begin{tabular}{|c|c|c|c|c|c|c|c|c|}
\hline & $\begin{array}{l}\text { Fid } \\
\text { (EvM) }\end{array}$ & $\begin{array}{l}\text { *Simult } \\
\text { Suspense }\end{array}$ & *Dépas & $\begin{array}{l}\text { Max } \\
\text { (Int) }\end{array}$ & $\begin{array}{l}\text { Fid } \\
(\text { ActP) }\end{array}$ & $\begin{array}{l}\text { Max } \\
\text { (Émo) }\end{array}$ & $\begin{array}{l}\text { Max } \\
\text { (Comp) }\end{array}$ & $\begin{array}{l}\text { Fid } \\
\text { (Act) }\end{array}$ \\
\hline $\begin{array}{l}\text { Essai } \\
+ \text { Journaliste } \\
\text { - Expert }\end{array}$ & *! & $(*)$ & & & * & & * & * \\
\hline $\begin{array}{l}\text { Jeu rapide } \\
+ \text { Journaliste } \\
\text { - Expert }\end{array}$ & & & *! & * & & & $*$ & \\
\hline $\begin{array}{l}\text { Ralenti } \\
\text { - Journaliste } \\
+ \text { Expert }\end{array}$ & & & & & & & $* !$ & \\
\hline
\end{tabular}

Tableau 1. Exemples d'interactions entre contraintes: essai, jeu rapide, ralenti

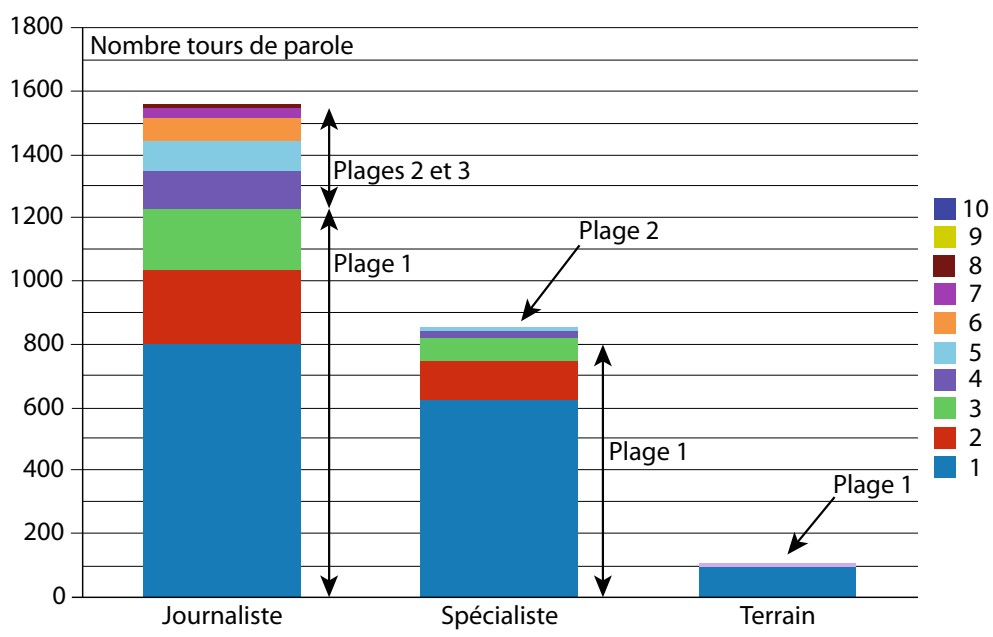

Rythme du jeu : nombre d'actions / 5 secondes

Figure 7. Interventions du journaliste et du spécialiste (consultant) en fonction des plages de rythme. Les couleurs représentent chacune un nombre d'actions par fenêtre de 5 secondes (1 à 10)

le journaliste du consultant, c'est le fait que le journaliste intervient aussi, même si c'est de manière beaucoup moins fréquente, dans les plages 2 et 3 , tandis que l'expert, lui, n'intervient pratiquement que dans la plage $\mathrm{I}$, jamais dans la plage 3 . Ces résultats montrent que le rythme du contenu commenté influe sur l'organisation du discours et notamment ici sur la répartition de la parole, sans doute en fonction des compétences linguistiques des locuteurs, c'est-à-dire leur capacité à parler sous contraintes. Le journaliste semble plus à même de produire du commentaire quand les contraintes amenées par le rythme du jeu sont plus fortes. 

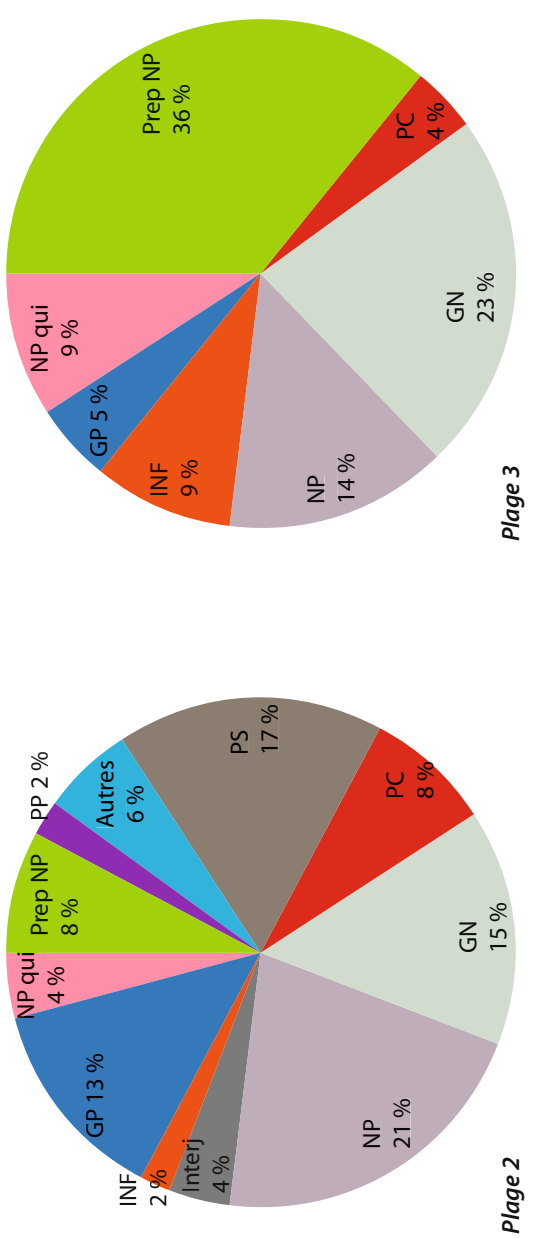

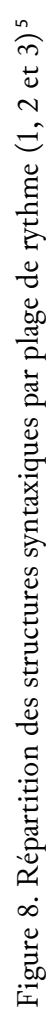

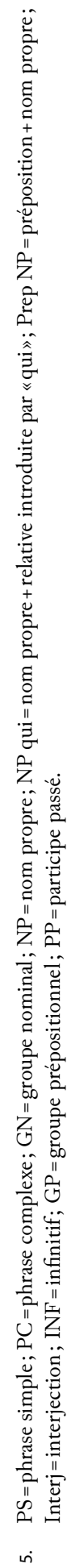

URL: http://discours.revues.org/9082 
Nous avons développé dans les sections précédentes l'idée que le style, en tant que "sélection opérée dans les moyens de la langue - moyens lexicaux, phraséologiques et grammaticaux» (Bakhtine, 1984:265), résultait d'un ensemble de contraintes, et que parmi celles-ci les contraintes matérielles de production ne devaient pas être écartées au profit des seules contraintes socio-pragmatiques habituellement identifiées. Cette dimension socio-pragmatique, que nous avons couverte sous l'appellation «contraintes de genre», ne suffit pas, pour un certain nombre de genres de discours, à expliquer la sélection des structures. Dans l'autre sens, le genre de discours peut être dans certains cas caractérisé par des structures linguistiques qui lui seraient propres. Ainsi, de nombreuses études du genre du commentaire sportif en direct, puisque c'est celui-ci qui nous intéresse, tant prosodiques (Audrit et al., 2012; Pršir et al., 2013) que syntaxiques (Deulofeu, 2000; Krazem, 20II ; Augendre et Mathon, zoI2a et b), ont cherché à montrer les structures spécifiques à ce genre, phonostyles ou constructions syntaxiques. Avec cette caractérisation du genre par les structures dites «spécifiques», se pose la question de ce qui est représentatif du genre. Ainsi, une analyse syntaxique d'un commentaire sportif en direct d'un sport autre qu'un match, comme par exemple une course de relais (4 X Ioo mètres), montre des constructions syntaxiques très différentes de celles observées dans les études mentionnées précédemment (voir Augendre et al., 20I5). Dans le même temps, un test de perception, portant sur la détection du sport sur la base des seuls indices prosodiques, montre qu'il est possible de distinguer le commentaire sportif du commentaire d'autres événements médiatiques, sans avoir accès aux structures syntaxiques ou au lexique (Mathon, 20I4). Enfin, un second test de perception, pratiqué dans les mêmes conditions de filtrage de l'information lexicale que le premier, montre que si les contraintes du commentaire en direct «play-by-play» sont très similaires en termes de rythme ou d'émotion véhiculée, alors les sujets ne distinguent pas le commentaire sportif du commentaire d'autres 
événements médiatiques (voir Augendre et al., 20I5). Par défaut, les sujets classent le «play-by-play» des autres événements médiatiques dans la catégorie commentaire sportif. Ainsi, plus que les structures syntaxiques ou le choix lexical, il semble que ce qui est représentatif du commentaire sportif ce sont des caractéristiques prosodiques découlant directement des contraintes matérielles de production, «Fid(Timing)», ainsi que des contraintes de genre: la contrainte «Fid(Événement)», qui s'applique au niveau du commentaire télévisé en direct, indépendamment du caractère sportif ou non, et «Max(Émo)» qui est appliquée au niveau du discours médiatique. Si les contraintes à l'origine de la production des styles ont été oubliées, pour autant les styles sont devenus représentatifs du genre et de ce fait deviennent des contraintes du genre, au sens où ce sont ces styles représentatifs du genre qui sont attendus de l'auditeur ou du téléspectateur, et qui feront qu'il reconnaitra le commentaire sportif comme étant «bien» produit. Nous pouvons alors considérer que le style, résultat des contraintes de production, s'institutionnalise et ce faisant devient une contrainte du genre. Pour illustrer notre propos, nous allons prendre l'exemple d'un autre discours très contraint, écrit, qui présente des spécificités syntaxiques intéressantes, celui des petites annonces. Celles-ci diffusées autrefois uniquement dans la presse écrite étaient payantes, le mode de rémunération se faisant à la ligne ou au nombre de caractères. Cette circonstance matérielle de production du discours amène une contrainte sur la taille du discours (il doit être bref) et implique une adaptation de la syntaxe qui explique par exemple l'absence systématique de déterminants devant les noms communs, ou de clitiques sujets devant les formes verbales. Si l'on observe une petite annonce publiée gratuitement sur un site en ligne (leboncoin.fr), qui n'impose aucune contrainte de taille, qui permet même l'édition de photos, on s'aperçoit que l'en-tête

[2] Appartement, $58 \mathrm{~m}^{2}$, rénové, $3^{\mathrm{e}}$ sans ascenseur, résidence calme, bien située. Proche gare (ร', côté Plessis Bouchard), commerces prox.

présente les caractéristiques syntaxiques d'une petite annonce classique. Pourtant, les circonstances matérielles à l'origine de ces structures spécifiques ont disparu. Ces dernières se sont institutionnalisées, de manière à former des contraintes de genre, qui permettent la reconnaissance d'un discours correspondant comme appartenant au genre «petite annonce».

\section{Conclusion}

Le travail d'analyse linguistique à plusieurs niveaux - prosodique, syntaxique et lexical - sur un genre de discours particulier, le commentaire sportif en direct, nous amène à rediscuter les dimensions définitoires du genre en tant qu'outils permettant d'expliquer la variété des styles présents dans ce type de discours. Si la notion de contraintes apparaît régulièrement dans la littérature sur le genre, celles-ci ne sont pas pour autant formalisées et leur influence sur le discours n'est pas directement mesurée. Par ailleurs, les circonstances matérielles de production du discours, si elles sont mentionnées comme étant une dimension définitoire, sont en réalité peu prises 
en compte, au profit de la dimension socio-pragmatique. Pourtant, pour le discours qui nous intéresse ici, la dimension matérielle est particulièrement importante, nous l'avons montré. Ainsi, notre objet d'étude nous a amenés à considérer le genre de discours, non pas comme un hyper-objet, caractérisé par différentes dimensions ou différents traits, qui aurait des structures linguistiques données, mais plutôt comme un set de contraintes, auxquelles s'ajoutent les contraintes liées aux circonstances matérielles et dans notre cas au contenu du commentaire (l'événement sportif). Ces contraintes ont été formalisées en utilisant la théorie de l'optimalité, ce qui nous permet d'examiner l'influence de chacune de ces contraintes sur le discours.

La nécessité de prendre en compte les contraintes extralinguistiques, pour l'analyse du commentaire sportif en direct, nous a amenés également à repenser notre méthodologie de travail. En effet, les travaux antérieurs sur le commentaire sportif en direct, que ce soit en prosodie ou en syntaxe, portent sur le discours uniquement, plus précisément sur la transcription orthographique du commentaire. Or, l'annotation des différents locuteurs qui construisent le commentaire, tout comme la segmentation en périodes (au sens de la linguistique textuelle, Adam, 2008) descriptives et explicatives, n'ont pas fourni un niveau d'analyse suffisamment fin pour rendre compte des constructions. Ainsi, sur la base des contraintes matérielles identifiées, l'analyse des structures linguistiques devait être précédée d'une annotation du contenu commenté, ce qui nous a amenés à annoter la vidéo du match de rugby qui nous a servi de corpus test. Ce travail d'annotation, fait manuellement, même s'il s'avère coûteux, offre des perspectives d'analyse et d'explication par rapport à la répartition de la parole, au choix des structures syntaxiques et du lexique, aux stratégies des locuteurs pour produire un discours sous contraintes.

L'annotation du corpus test nous a permis de juger de la faisabilité d'une telle démarche. Mais nous l'avons vu, si les sets de contraintes que nous avons identifiés pour le commentaire sportif en direct sont stables, en revanche l'application de ces contraintes dépend beaucoup du sport. Nous avons donné quelques illustrations sur la base d'autres sports, nous allons procéder au même type d'annotation des images, afin de vérifier l'application de nos contraintes sur le commentaire d'autres sports: ceci devrait nous permettre de donner une analyse plus représentative du commentaire sportif en général, qui ne serait pas réduite aux commentaires de matchs de football.

Enfin, le genre de discours pose la question de la représentation qu'en ont les locuteurs et surtout les récepteurs. Quels sont les paramètres qui font que le commentaire sportif est reconnu en tant que tel? Quels sont les paramètres qui font qu'un commentaire sportif est jugé comme un «bon» ou un «mauvais» commentaire? $\mathrm{Au}$-delà de la dimension thématique, qui influe évidemment sur la reconnaissance du genre, et de la qualité des connaissances encyclopédiques du journaliste, qui en font un bon ou un mauvais commentateur, trouve-t-on d'autres paramètres pertinents? La dimension orale du commentaire sportif en direct, et la possibilité de travailler sur des paramètres extralexicaux, fournissent des pistes intéressantes de recherche sur cette dernière question. 


\section{Références}

AdAm, J.-M. 2008. La linguistique textuelle. Introduction à l'analyse textuelle des discours. Paris: A. Colin [2 $2^{\mathrm{e}}$ éd.].

Audrit, S. et al. 20I2. Sport in the Media: A Contrasted Study of Three Sport Live Media Reports with Semi-automatic Tools. In M. Qiuwu, D. Hongwei et D. Hirst (éd.), Proceedings of the 6th International Conference on Speech Prosody. Shanghai: Tongji University Press: I27-I30. Vol. I. En ligne à l'adresse suivante: http://sprosig.isle. illinois.edu/sp2or2/pageı $57 . h t m l$.

AugEndRe, S. et al. 20I4. Influence des contraintes extralinguistiques sur le discours: cas du commentaire sportif télévisé. In F. Neveu et al. (éd.), $4^{e}$ congrès mondial de Linguistique française - CMLF 2014 (Berlin, I9-23 juillet 20I4). Les Ulis: EDP Sciences: 19051924. En ligne à l'adresse suivante: http://www.shs-conferences.org/articles/shsconf/ pdf/20I4/05/shsconf_cmlfi4_or38I.pdf.

Augendre, S. et al. 2015 (soumis). Live TV Sports Commentaries: Specific Syntactic Structures and General Constraints. In Journée ConSciLa: «Grammaire des genres et des styles: quelles approches privilégier?» (Paris, I6 janvier 2015).

Augendre, S. et Mathon, C. 2orza. Comment l'information à transmettre influencet-elle les constructions référentielle et syntaxique du commentaire sportif en direct? In F. Neveu et al., $3^{e}$ congrès mondial de Linguistique française - CMLF 2012 (Lyon, 4-7 juillet 20I2). Les Ulis: EDP Sciences: 2027-2040. En ligne à l'adresse suivante : http://www.shs-conferences.org/articles/shsconf/pdf/2OI2/oI/shsconf_cmlfi2_ oo0274.pdf.

Augendre, S. et Mathon, C. 20i2b. Construction of Referents in a Corpus of Live Sports Comment: Informational Structure, Syntactic and Prosodic Realization. In H. Mello, M. Pettorino et T. Raso (éd.), Proceedings of the VIIth GSCP International Conference. Speech and Corpora. Florence: Firenze University Press : 197-20I.

Bakhtine, M. M. 1984. Esthétique de la création verbale. Paris: Gallimard. Chap.: Les genres du discours: $263-308$.

BRES, J. 2007. Le discours médiatique sportif aux prises avec le temps verbal. In M. Вrотн et al. (éd.), Le français parlé des médias: actes du colloque de Stockbolm, 8-I2 juin 2005. Stockholm: Université de Stockholm: 83-96.

CAmpbell, N. 2008. Individual Traits of Speaking Style and Speech Rhythm in a Spoken Discourse. In A. Esposito (éd.), Verbal and Nonverbal Features of Human-Human and Human-Machine Interaction. Berlin: Springer: I07-I20.

CANVAT, K. 1999. Enseigner la littérature par les genres: pour une approche théorique et didactique de la notion de genre littéraire. Bruxelles - Paris: De Boeck - Duculot.

Charaudeau, P. 2002. Visées discursives, genres situationnels et construction textuelle. In M. Ballabriga (éd.), Analyse des discours. Types et genres: communication et interprétation. Toulouse : Éditions universitaires du Sud: $45^{-73}$.

Deulofeu, J. 2000. Les commentaires sportifs constituent-ils un «genre», au sens linguistique du terme? In M. BILGER (éd.), Corpus: méthodologie et applications linguistiques. Paris - Perpignan: Champion - Presses universitaires de Perpignan: 27I-295. 
Goldman, J.-P., Auchlin, A. et Simon, A. C. 2oir. Discrimination de styles de parole par analyse prosodique semi-automatique. In H.-Y. Yoo et E. Delais-Roussarie (éd.), Actes de la conférence «Interface, discours et prosodie»-IDP 2009 (Paris, 9-II septembre 2009). 207-22I. En ligne à l'adresse suivante: http://makino.linguist.jussieu.fr/idpog/docs/ IDP_actes/Articles/Goldman.pdf.

Hartmann, C. 2oir. Pre-fabricated Speech Formulas as Long-term Memory Solutions to Working Memory Overload. Thèse de doctorat. Université de Zurich.

KRAZEM, M. 20II. Représenter les relations entre grammaire et genres de discours : exemple des commentaires sportifs. $\operatorname{Linx} 64-65: 45^{-68 .}$

Lortal, G. et Mathon, C. 2008. Motion and Emotion or How to Align Emotional Cues with Game Actions. In A. Khurshid (éd.), Sentiment Analysis: Emotion, Metaphor, Ontology and Terminology - EMOT 2008 (27 mai 2008). Dublin: Trinity College: 79-85. En ligne à l'adresse suivante: http://www.lrec-conf.org/proceedings/lrec2oo8/ workshops/W6_Proceedings.pdf.

Mathon, C. 20i4. Perception des phonostyles et représentativité du phonogenre: le cas du commentaire sportif en direct. Nouveaux cabiers de linguistique française 31 : 93-103.

Mathon, C. et Boulakia, G. 2009. Le commentaire sportif en direct: une combinatoire de différentes fonctions de la prosodie. In H.-Y. Yoo et E. Delais-Roussarie (éd.), Actes de la conférence "Interface, discours et prosodie»-IDP 2009 (Paris, 9-II septembre 2009). 287-30I. En ligne à l'adresse suivante: http://makino.linguist.jussieu.fr/idpog/docs/ IDP_actes/Articles/mathon.pdf.

Mouchet, A., Uhlrich, G. et Bouthier, D. 2005. La continuité du jeu en rugby: théorie et méthodologie d'une étude comparative entre nations. Impulsions 4: 79-99.

Prince, A. et Smolensky, P. 1993. Optimality Theory: Constraint Interaction in Generative Grammar. Malden - Oxford - Victoria: Blackwell Publishing.

Pršir, T., Goldman, J.-P. et Auchlin, A. 20I3. Variation prosodique situationnelle: étude sur corpus de huit phonogenres en français. In P. Mertens et A. C. Simon (éd.), Proceedings of the Prosody-Discourse Interface Conference - IDP 2013 (Louvain, II-I3 septembre 20I3). IO7-II2. En ligne à l'adresse suivante: http://www.arts.kuleuven. be/ling/franitalco/conference/idp2or3/documents/proceedings_idp2or3.

Richer, J.-J. 20II. Les genres de discours: une autre approche possible de la sélection de contenus grammaticaux pour l'enseignement/apprentissage du FLE? Linx 64-65: 15-26. 